Please do not remove this page

RMIT

UNIVERSITY

\title{
A compact technique for allocation of coefficient to an optical signal
}

Bui, Lam; Mansoori, Sana; Mitchell, Arnan

https://researchrepository.rmit.edu.au/esploro/outputs/9921862592101341/filesAndLinks?institution=61RMIT_INST\&index=null

Bui, L., Mansoori, S., \& Mitchell, A. (2006). A compact technique for allocation of coefficient to an optical signal. Proceedings of the 31st Australian Conference on Optical Fibre Technology and Australian Optical Society (ACOFT\&AOS 2006). https://doi.org/10.1109/ACOFT.2006.4519259

Published Version: https://doi.org/10.1109/ACOFT.2006.4519259

Repository homepage: https://researchrepository.rmit.edu.au

(c) 2006 IEEE. Personal use of this material is permitted. However, permission to reprint/republish this material for advertising or promotional purposes or for creating new collective works for resale or redistribution to servers or lists, or to reuse any copyrighted component of this work in other works must be obtained from the IEEE.

Downloaded On 2023/04/27 01:30:53 +1000 


\title{
A compact technique for allocation of coefficient to an optical signal
}

\author{
Lam Anh Bui ${ }^{1}$, Sana Mansoori ${ }^{2}$, Arnan Mitchell ${ }^{1}$ \\ ${ }^{1}$ School of Electrical and Computer Engineering, RMIT University, \\ GPO Box 2476V, Melbourne 3001, Australia \\ ${ }^{2}$ Department of Electrical and Electronics Engineering, College of Engineering, University of Bahrain
}

\begin{abstract}
A novel and compact scheme to assign coefficient to a wavelength channel is presented. This scheme realises both positive and negative coefficients over the full range from -1 to 1 . A proof of concept is demonstrated for RF frequencies ranging from 1 to $20 \mathrm{GHz}$. This scheme allows implementation of transversal filters with arbitrary responses.
\end{abstract}

\section{INTRODUCTION}

Modern defense receivers require signal processors that are broad band and reconfigurable [1]. Transversal signal processors are very flexible and can be adapted to several responses dynamically by reconfiguring tap coefficients [1]. For broadband performance, these filters are often implemented using microwave photonic techniques.

A number of photonic transversal filter architectures have been demonstrated in the last decade [1]. Most of these implementations utilize non-coherent detection in which tap coefficients are proportional to optical power. This consequently restricts the coefficients to positive values only which also limits the range of possible synthesized responses. To overcome this limitation, a number of methods to achieve both positive and negative coefficients for transversal filters have been proposed, including 1) differential detection, 2) inversion within a gain medium and 3) dual modulators with opposite driving or dual input/output Mach Zehnder modulator [1, 2]. Although these techniques address the problem of single signed coefficients and allow synthesizing arbitrary filter response, they are restricted to allocation of a fixed sign coefficient to a given wavelength and do not allow the possibility to dynamically change the coefficient sign. This therefore reduces the flexibility to reconfigure the system response.

In this paper, we present a novel technique that allows arbitrary allocation of a signed coefficient to a wavelength channel. This coefficient can be altered rapidly, and hence allows fast and arbitrary reconfiguration of the system response. The organization of this paper is as follows. In Section II, the novel concept is described and its principle of operation is explained. Section III presents a proof of concept experiment demonstrating continuous variation of the coefficient over the full range of -1 to 1 for RF signal from 1 to $20 \mathrm{GHz}$. Section IV presents the experimental results and Section V discusses the extension of the concept to multiple wavelength channels and other improvements of the proposed scheme. Finally, the paper is concluded in Section VI.

\section{PRINCIPLE OF OPERATION}

The novel approach is based on the selective modulation of optical wavelengths using Mach Zehnder modulators (MZM) biased at positive or negative quadrature. This configuration is schematically presented in Figure 1.

The proposed scheme consists of two MZMs driven simultaneously through a $3 \mathrm{~dB}$ RF coupler. These modulators are biased at positive and negative quadrature points as indicated in Figure 1. The optical input and output of the MZMs are combined using optical polarization beam splitters (PBS) to avoid coherent interference. Using this configuration, the optical power is split between the two modulating arms according to its polarisation. At the photodetector, two orthogonal polarization components which are modulated with opposite signs are converted to RF signals with their respective signs and weights. The resulting RF signal is the sum of these components and thus takes the dominant component sign. In other word, by controlling just the polarization, an optical wavelength can be assigned an arbitrary weight from -1 (polarized horizontally) through 0 (polarized at 45 degrees) then to +1 (polarized vertically).

To implement this scheme, a method to dynamically manipulate the signal polarization. It has been demonstrated that an acousto-optic polarization coupling (AOPC) device rotates polarization for a narrow band of optical wavelength that phase matches with the acoustic frequency [3]. The angle of rotation is dictated by the acoustic signal strength and can be arbitrarily controlled [3]. In this paper the concept presented in Figure 1 is demonstrated using an AOPC as a polarization rotator. 


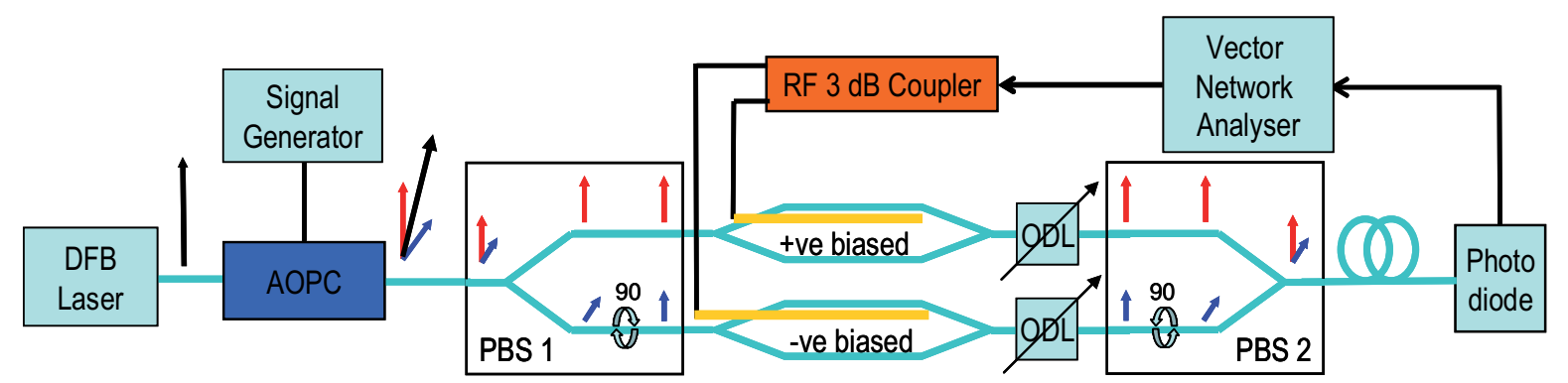

Figure 1: Coefficient allocation principle and the proof of concept experimental setup
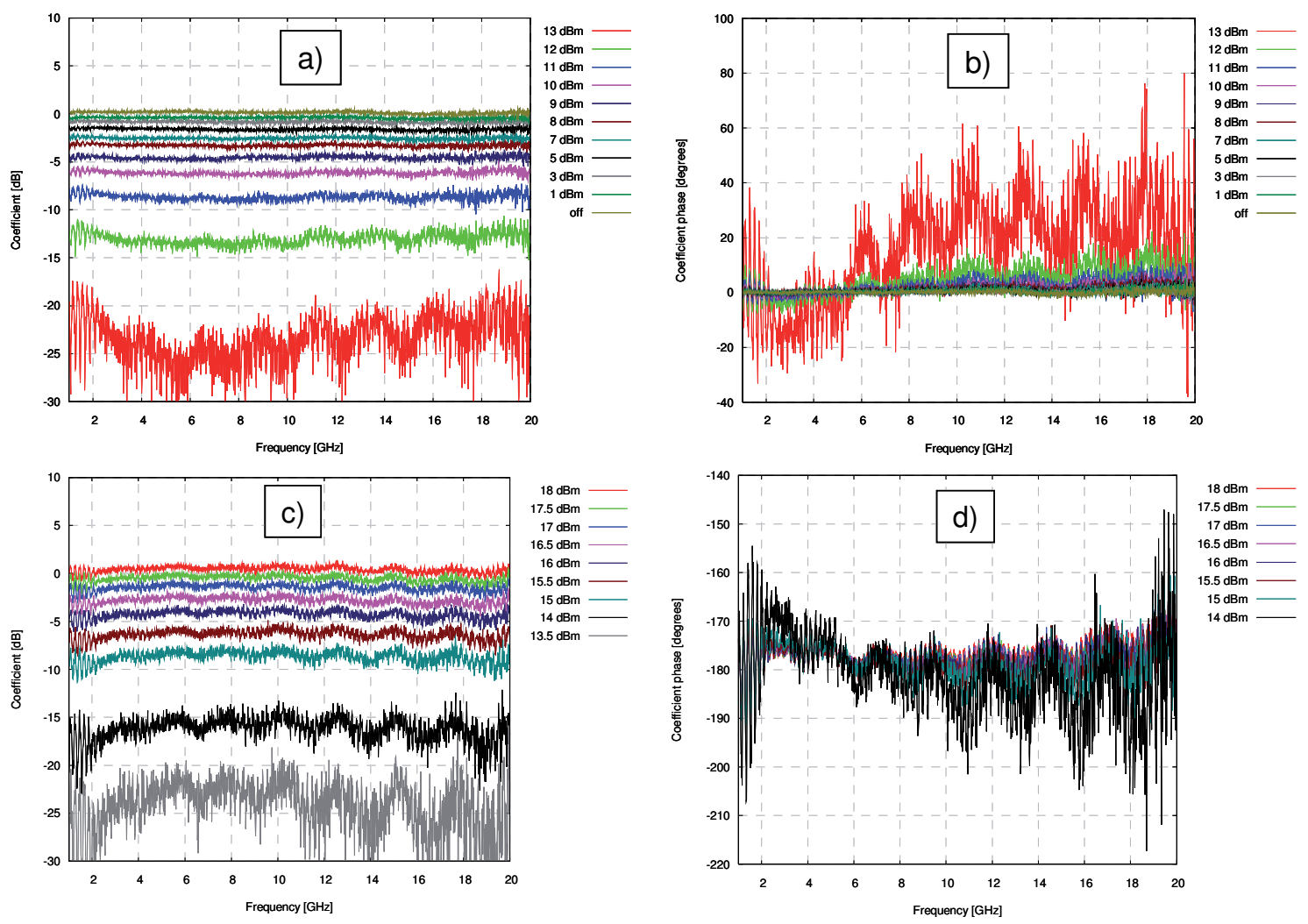

Figure 2: Measured system coefficient at various acoustic power level settings: positive coefficients a) magnitude, b) phase, and Negative coefficients c) magnitude, d) phase.

\section{PROOF OF CONCEPT EXPERIMENT}

The experimental setup to demonstrate the proposed scheme is presented in Figure 1. Two MZMs with polarization maintaining fibre pigtails were combined optically at the input and output using polarization beam splitters (PBS 1 and PBS 2). The PBSs were designed such that one of the two output arms had a built in 90 degree fibre rotation so that the output polarization was aligned with the MZM input polarization as illustrated in Figure 1. The modulators were biased at positive and negative quadrature points respectively. RF signals were split equally between the two modulators through a broadband $3 \mathrm{~dB}$ microwave coupler. Variable optical delay lines were used between the MZMs and the PBS 2 to match the electrical lengths between the two modulating arms. This matching was performed by measuring the phase response of each individual arm with a vector network analyzer (VNA). The system was output to a broadband photo-detector where the optical signals were demodulated and summing of the two components took place.

In this experiment, a DFB laser was used to provide the optical carrier. This optical carrier had a $1555 \mathrm{~nm}$ wavelength and was polarized vertically as shown in Figure 1. Rotation of the carrier polarization was controlled by an acousto-optic polarization coupler (AOPC). This AOPC was designed and fabricated in Lithium Niobate at RMIT University using the method reported in [3]. Characterisation of the AOPC shows that the device exhibited phase matching to $1555 \mathrm{~nm}$ wavelength at an acoustic frequency of $171.344 \mathrm{MHz}$. The AOPC wavelength drift was also minimized through maintaining the device at $25^{\circ} \mathrm{C}$ during operation. 


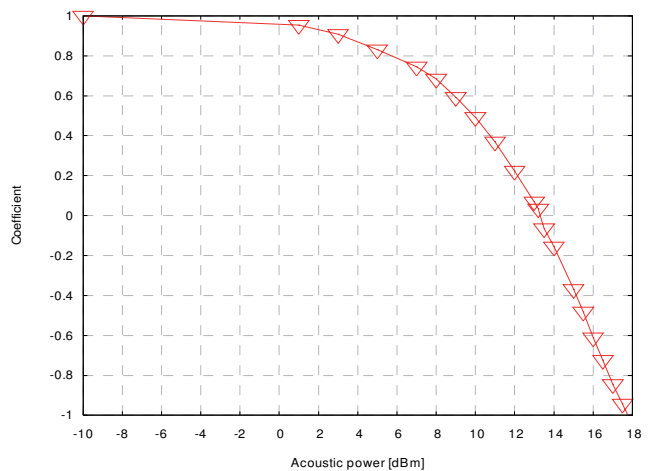

Figure 3: Measured coefficient at $10 \mathrm{GHz}$ as a function of acoustic power.

A vector network analyzer was used to measure the system response. The VNA was calibrated to the system response when the acoustic signal driving the AOPC is turned off. In this state, the AOPC behaved like a simple optical waveguide and did not alter the carrier polarization. The un-rotated carrier is thus routed to the positive biased MZM and the system thus exhibits a coefficient of 1 . In a similar fashion, the system response was then measured for each increment of the acoustic power, starting with the acoustic power of -10 $\mathrm{dBm}$ where little polarisation is converted and finishing at the power of $18 \mathrm{dBm}$ when full conversion is attained. At the full polarization conversion, the optical carrier was polarized vertically. It is thus modulated only by the negative biased MZM. The system therefore represents the most negative coefficient of -1 .

\section{EXPERIMENTAL RESULTS}

Figures 2 a)-d) present both the magnitude and the phase of these measurements. Figures 2 a) and b) presents the measured magnitude and phase of the coefficient between 1$20 \mathrm{GHz}$ when the acoustic power varied from completely off to $13 \mathrm{dBm}$. It is evident that a continuous variation of the coefficient magnitude from below $-20 \mathrm{~dB}$ to $0 \mathrm{~dB}$ is possible. The magnitude response is flat within $+/-0.5 \mathrm{~dB}$ and the phase response maintains around 0 degree across the whole measurement band. For small coefficient value such as -20 $\mathrm{dB}$, the signal approaches the setup noise floor which increases the uncertainty in the phase measurements.

Further increasing the acoustic power results in flipping of coefficient sign from positive to negative as evident in Figures $2 \mathrm{c}$ ) and d). At $18 \mathrm{dBm}$, a complete polarization conversion is achieved and the coefficient of -1 is attained. A continuous variation of the coefficient magnitude from $-20 \mathrm{~dB}$ to $0 \mathrm{~dB}$ is also achievable over the complete frequency band. The coefficient phase always maintains at 180 degree in all measurements, which signifies they are negative.

It is evident that the coefficient is frequency independent over the complete measured band. It is therefore useful to plot the coefficient value against the acoustic power. Figure 3 presents this result for coefficient determined at $10 \mathrm{GHz}$. From Figure 3, it is concluded that an arbitrary coefficient can be assigned to an optical wavelength by driving the AOPC at a suitable power level.

\section{DISCUSSIONS}

The scheme presented in Figure 1 is demonstrated for a single optical wavelength. The AOPC has been demonstrated for multiple channel operation [4], it is thus straight forward to extend the presented concept to a number of wavelength channels. The number of wavelength channels that can be simultaneously operated would be determined by the AOPC. It is anticipated that up to 8 wavelength channels can be operated at once [4]. In such configuration, each wavelength channels could be individually assigned a coefficient. This flexibility allows realization of a transversal filter using only a single device. Additionally, the system capability to provide both positive and negative coefficients enable construction of arbitrary filter responses which are not possible with positive only coefficient systems [1].

Another advantage of the proposed scheme is its compactness. Further reduction of the device implementation can be achieved by replacing two MZMs by a dual input MZM using similar arrangement presented in [1]. Complementary values of the coefficient would be also available when a single output MZM is replaced by a dual output MZM. Finally, the complete scheme could be fabricated monolithically in Lithium Niobate to realise a single chip transversal signal processor. It is anticipated that in such integration, the acoustic design would be critical to the device's stability and flexibility.

\section{CONCLUSIONS}

A compact and novel scheme to assign coefficient to an optical wavelength channel is proposed. The scheme is capable to realise for both positive and negative coefficient and covers the complete range of -1 to 1 . The proof of concept experiment shows that the coefficient is continuously variable and flat across the $1-20 \mathrm{GHz}$ range. It is also proposed that the presented scheme could be extended to several wavelength channels. This allows realization of broad band transversal filters with arbitrary responses.

\section{ACKNOWLEDGEMENT}

The authors wish to acknowledge the technical assistance of the RMIT University MMTC staff.

\section{REFERENCES}

[1] J. Capmany, B. Ortega, and D. Pastor, "A tutorial on microwave photonic filters," Lightwave Technology, Journal of, vol. 24, pp. 201-229, 2006.

[2] R. A. Minasian, "Photonic signal processing of microwave signals," Microwave Theory and Techniques, IEEE Transactions on, vol. 54, pp. 832-846, 2006.

[3] H. Mendis, A. Mitchell, I. Belski, M. Austin, and O. A. Peverini, "Design, realisation and analysis of an apodised, filmloaded acousto-optic tunable filter," Applied Physics B: Lasers and Optics, vol. 73, pp. 489 - 493, 2001.

[4] K. W. Cheung, D. A. Smith, J. E. Baran, and B. L. Heffner, "Multiple channel operation of integrated acousto-optic tunable filter," Electronics Letters, vol. 25, pp. 375-376, 1989. 